Article

\title{
On the Existence of the Solutions of a Fredholm Integral Equation with a Modified Argument in Hölder Spaces
}

\author{
Merve Temizer Ersoy* $*$ and Hasan Furkan \\ Institute of Science and Technology, Kahramanmaraş Sütçü İmam University, Kahramanmaraş 46100, Turkey; \\ hasanfurkan@gmail.com \\ * Correspondence: mervetemizer@hotmail.com
}

Received: 29 September 2018; Accepted: 16 October 2018; Published: 19 October 2018

check for updates

\begin{abstract}
This article concerns the entity of solutions of a quadratic integral equation of the Fredholm type with an altered argument, $x(t)=p(t)+x(t) \int_{0}^{1} k(t, \tau)(T x)(\tau) d \tau$, where $p, k$ are given functions, $T$ is the given operator satisfying conditions specified later and $x$ is an unknown function. Through the classical Schauder fixed point theorem and a new conclusion about the relative compactness in Hölder spaces, we obtain the existence of solutions under certain assumptions. Our work is more general than the previous works in the Conclusion section. At the end, we introduce several tangible examples where our entity result can be adopted.
\end{abstract}

Keywords: Fredholm integral equation; Schauder fixed point theorem; Hölder condition

\section{Introduction}

The work of differential equations, with an altered argument being latest, has continued for decades. For more data and consequences related to these equations, see [1-3]. These topics have linear modifications of their arguments and have been worked on by the authors in the papers [1-15]. Integral equations of course stem from several applications in specification numerous real-world problems (see $[16,17]$ and the references therein). Quadratic integral equations arise naturally in applications to real-world problems. For example, problems in the kinetic theory of gases and in the theory of radiative transfer lead to the quadratic integral equation:

$$
x(t)=1+t x(t) \int_{0}^{1} \frac{\Phi(\tau)}{t+\tau} x(\tau) d \tau,
$$

(see [18-21]). The integral equations of a similar form have been examined by several authors [22-28]. Furthermore, some studies using similar techniques have been dedicated to a micropolar porous body and vibrations in thermoelasticity $[29,30]$.

Very recently, J. Banaś and R. Nalepa et al. [4] studied the following equation:

$$
x(t)=p(t)+x(t) \int_{a}^{b} k(t, \tau) x(\tau) d \tau .
$$

Further, J. Caballero, M. Darwish and K. Sadarangani et al. [5] studied the following equation:

$$
x(t)=p(t)+x(t) \int_{0}^{1} k(t, \tau) x(r(\tau)) d \tau
$$


Furthermore, J. Cabelloro Mena, R. Nalepa and K. Sadarangani et al. [6] studied the following equation:

$$
x(t)=p(t)+x(t) \int_{0}^{1} k(t, \tau)\left\{\max _{\eta \in[0, r(\tau)]}|x(\eta)|\right\} d \tau .
$$

The purpose of this paper is to examine the existence of solutions of the following integral equation of the Fredholm type with a changed argument,

$$
x(t)=p(t)+x(t) \int_{0}^{1} k(t, \tau)(T x)(\tau) d \tau, \quad t \in I=[0,1]
$$

Equation (4) is more general than many equations considered up to now and includes (1), (2) and (3) as special cases. Notice that Equation (1) in [4] for $a=0$ and $b=1$ is a particular case of (4) with $(T x)(\tau)=x(\tau)$. Furthermore, it should be noted that Equation (4) is more general than Equation (2) considered in [5]. If we take $(T x)(\tau)=x(r(\tau))$, then the equation:

$$
x(t)=p(t)+x(t) \int_{0}^{1} k(t, \tau) x(r(\tau)) d \tau
$$

is obtained from Equation (4). Further, notice that Equation (3) in [6] is a particular case of (4), for $(T x)(\tau)=\max _{\eta \in[0, r(\tau)]}|x(\eta)|$, where the function $r:[0,1] \rightarrow[0,1]$ is continuous and nondecreasing.

Compared to the previous works [4-6], we have further generalized the new assumptions in finding the solutions of (1), (2) and (3).

Our solutions substitute for the spaces of functions satisfactory the Hölder condition, and this is a source of the originality of the article. To do this, we will use a recent consequence about the classical Schauder fixed point theorem and the relative compactness in Hölder spaces.

\section{Preliminaries and Notations}

In this section, we present definitions, notations and theorems that are used along this paper. The following known definitions are available in [4,5,31,32].

Let $[a, b]$ be a closed interval in $\mathbb{R}$; by $C[a, b]$, we indicate the space of continuous functions defined on $[a, b]$ equipped with the supremum norm, i.e.,

$$
\|x\|_{\infty}=\sup \{|x(t)|: t \in[a, b]\}
$$

for $x \in C[a, b]$. For a fixed $\alpha$ with $0<\alpha \leq 1$, by $H_{\alpha}[a, b]$, we will indicate the spaces of the real functions $x$ defined on $[a, b]$ and satisfying the Hölder condition, that is those functions $x$ for which there exists a constant $H_{x}^{\alpha}$ such that:

$$
|x(t)-x(s)| \leq H_{x}^{\alpha}|t-s|^{\alpha}
$$

for all $t, s \in[a, b]$. It is well proven that $H^{\alpha}[a, b]$ is a linear subspace of $C[a, b]$. Furthermore, for $x \in H^{\alpha}[a, b]$, by $H_{x}^{\alpha}$, we will indicate the least possible stable value for which Inequality (5) is satisfied. Rather, we put:

$$
H_{x}^{\alpha}=\sup \left\{\frac{|x(t)-x(s)|}{|t-s|^{\alpha}}: t, s \in[a, b] \text { and } t \neq s\right\} .
$$

The space $H_{\alpha}[a, b]$ with $0<\alpha \leq 1$ may be equipped with the norm:

$$
\|x\|_{\alpha}=|x(a)|+H_{x}^{\alpha}
$$

for $x \in H_{\alpha}[a, b]$. Here, $H_{x}^{\alpha}$ is defined by (6). In [4], the authors demonstrated that $\left(H_{\alpha}[a, b]\right.$, $\left.\|\cdot\|_{\alpha}\right)$ with $0<\alpha \leq 1$ is a Banach space. 
Lemma 1. For $0<\alpha \leq 1$ and $x \in H_{\alpha}[a, b]$, we have:

$$
\|x\|_{\infty} \leq \max \left(1,(b-a)^{\alpha}\right)\|x\|_{\alpha} .
$$

In particular, the inequality $\|x\|_{\infty} \leq\|x\|_{\alpha}$ is satisfied for $a=0$ and $b=1$ [4].

Lemma 2. For $0<\alpha<\beta \leq 1$, we have:

$$
H_{\beta}[a, b] \subset H_{\alpha}[a, b] \subset C[a, b]
$$

Furthermore, for $x \in H_{\beta}[a, b]$, we have:

$$
\|x\|_{\alpha} \leq \max \left(1,(b-a)^{\beta-\alpha}\right)\|x\|_{\beta}
$$

Particularly, the inequality $\|x\|_{\infty} \leq\|x\|_{\alpha} \leq\|x\|_{\beta}$ is satisfied for $a=0$ and $b=1$, [4].

Lemma 3. Let us assume that $0<\alpha<\beta \leq 1$ and $E$ is a bounded subset in $H_{\beta}[a, b]$, then $E$ is a relatively compact subset in $H_{\alpha}[a, b][5]$.

Lemma 4. Assume that $0<\alpha<\beta \leq 1$, and by $B_{r}^{\beta}$, we indicate the ball centered at $\theta$ and radius $r$ in the space $H_{\beta}[a, b]$, i.e., $B_{r}^{\beta}=\left\{x \in H_{\beta}[a, b]:\|x\|_{\beta} \leq r\right\}$. $B_{r}^{\beta}$ is a closed subset of $H_{\alpha}[a, b][5]$.

Corollary 1. Assume that $0<\alpha<\beta \leq 1$ and $B_{r}^{\beta}$ is a relatively compact subset in $H_{\alpha}[a, b]$ and a closed subset of $H_{\alpha}[a, b]$, then $B_{r}^{\beta}$ is a compact subset in the space $H_{\alpha}[a, b],[5]$.

Now let us give the following theorem, which is the base tool used in our study.

Theorem 1 (Schauder's fixed point theorem). Let E be a nonempty, compact subset of a Banach space $(X,\|\cdot\|)$, convex, and let $T: E \rightarrow E$ be a continuity mapping. Then, $T$ has at least one fixed point in $E$ [7].

\section{Main Result}

Now, we are ready to give the main result of the paper. In this section, we introduce the following sufficient conditions for the main theorem in our study, and we will prove the solvability of Equation (4) in Hölder spaces.

Hereafter, we suppose unless stated otherwise that $\alpha$ and $\beta$ are arbitrarily fixed numbers such that $0<\alpha<\beta \leq 1$.

Theorem 2. Assume that the following Conditions (i)-(iv) are satisfied:

(i) $p \in H_{\beta}[0,1]$.

(ii) $k:[0,1] \times[0,1] \rightarrow \mathbb{R}$ is a continuous function such that there exists a constant $k_{\beta}>0$ such that:

$$
|k(t, \tau)-k(s, \tau)| \leq k_{\beta}|t-s|^{\beta},
$$

for any $t, s, \tau \in[0,1]$.

(iii) The operator $T: H_{\beta}[0,1] \rightarrow C[0,1]$ is continuous on $H_{\beta}[0,1]$ with respect to the norm $\|\cdot\|_{\alpha}$, and there exists a function $f: \mathbb{R}_{+} \rightarrow \mathbb{R}_{+}$, which is non-decreasing such that the inequality holds:

$$
\|T x\|_{\infty} \leq f\left(\|x\|_{\beta}\right)
$$

for any $x \in H_{\beta}[0,1]$. 
(iv) There exists a positive solution $r_{0}$ of the inequality:

$$
\|p\|_{\beta}+\left(2 K+k_{\beta}\right) r f(r) \leq r
$$

where the constant $K$ is defined by:

$$
\sup \left\{\int_{0}^{1}|k(t, \tau)| d \tau: t \in[0,1]\right\} \leq K
$$

Then, Equation (4) has at least one solution $x=x(t)$ belonging to space $H_{\alpha}[0,1]$.

Proof. Now, let us consider $x \in H_{\beta}[0,1]$ and the operator $F$ defined on the space $H_{\beta}[0,1]$ by the formula:

$$
(F x)(t)=p(t)+x(t) \int_{0}^{1} k(t, \tau)(T x)(\tau) d \tau,
$$

for $t \in[0,1]$. Then, for arbitrarily fixed $t, s \in[0,1],(t \neq s)$, in view of our assumptions, we get:

$$
\begin{aligned}
& (F x)(t)-(F x)(s) \\
= & p(t)+x(t) \int_{0}^{1} k(t, \tau)(T x)(\tau) d \tau-p(s)-x(s) \int_{0}^{1} k(s, \tau)(T x)(\tau) d \tau \\
= & p(t)-p(s)+x(t) \int_{0}^{1} k(t, \tau)(T x)(\tau) d \tau-x(s) \int_{0}^{1} k(s, \tau)(T x)(\tau) d \tau \\
& +x(s) \int_{0}^{1} k(t, \tau)(T x)(\tau) d \tau-x(s) \int_{0}^{1} k(t, \tau)(T x)(\tau) d \tau \\
= & p(t)-p(s)+(x(t)-x(s)) \int_{0}^{1} k(t, \tau)(T x)(\tau) d \tau \\
& +x(s) \int_{0}^{1}(k(t, \tau)-k(s, \tau))(T x)(\tau) d \tau
\end{aligned}
$$

and:

$$
\begin{aligned}
& \frac{|(F x)(t)-(F x)(s)|}{|t-s|^{\beta}} \\
\leq & \frac{|p(t)-p(s)|}{|t-s|^{\beta}}+\frac{|x(t)-x(s)|}{|t-s|^{\beta}} \int_{0}^{1}|k(t, \tau) \|(T x)(\tau)| d \tau \\
& +\frac{|x(s)|^{\beta}}{|t-s|_{0}^{1}|k(t, \tau)-k(s, \tau) \|(T x)(\tau)| d \tau} \\
\leq & H_{p}^{\beta}+\|x\|_{\beta}\|T x\|_{\infty} \int_{0}^{1}|k(t, \tau)| d \tau \\
& +|x(s)| \int_{0}^{1} \frac{|k(t, \tau)-k(s, \tau)|}{|t-s|^{\beta}}|(T x)(\tau)| d \tau \\
\leq & H_{p}^{\beta}+\|x\|_{\beta}\|T x\|_{\infty} K+\|x\|_{\infty}\|T x\|_{\infty} \int_{0}^{1} k_{\beta} \frac{|t-s|^{\beta}}{|t-s|^{\beta}} d \tau \\
\leq & H_{p}^{\beta}+\|x\|_{\beta}\|T x\|_{\infty} K+\|x\|_{\beta}\|T x\|_{\infty} k_{\beta} \\
\leq & H_{p}^{\beta}+\|x\|_{\beta} f\left(\|x\|_{\beta}\right) K+\|x\|_{\beta} f\left(\|x\|_{\beta}\right) k_{\beta} \\
= & H_{p}^{\beta}+\left(K+k_{\beta}\right)\|x\|_{\beta} f\left(\|x\|_{\beta}\right) .
\end{aligned}
$$

This demonstrates that the operator $F$ maps $H_{\beta}[0,1]$ into itself. 
Besides, for any $x \in H_{\beta}[0,1]$, we get:

$$
\begin{aligned}
|(F x)(0)| & \leq|p(0)|+|x(0)| \int_{0}^{1}|k(0, \tau)||(T x)(\tau)| d \tau \\
& \leq|p(0)|+\|x\|_{\infty}\|T x\|_{\infty} K \\
& \leq|p(0)|+\|x\|_{\beta}\|T x\|_{\infty} K \\
& \leq|p(0)|+\|x\|_{\beta} f\left(\|x\|_{\beta}\right) K .
\end{aligned}
$$

By the inequalities (7) and (8), we derive that:

$$
\|F x\|_{\beta} \leq\|p\|_{\beta}+\left(2 K+k_{\beta}\right)\|x\|_{\beta} f\left(\|x\|_{\beta}\right) .
$$

Since positive number $r_{0}$ is the solution of the inequality given in Hypothesis (iv), from (9) and function $f: \mathbb{R}_{+} \rightarrow \mathbb{R}_{+}$, which is non-decreasing, we conclude that the inequality:

$$
\|F x\|_{\beta} \leq\|p\|_{\beta}+\left(2 K+k_{\beta}\right) r_{0} f\left(r_{0}\right) \leq r_{0}
$$

holds. As a result, it follows that $F$ transform the ball:

$$
B_{r_{0}}^{\beta}=\left\{x \in H_{\beta}[0,1]:\|x\|_{\beta} \leq r_{0}\right\}
$$

into itself. That is, $F: B_{r_{0}}^{\beta} \rightarrow B_{r_{0}}^{\beta}$. Thus, we have that the set $B_{r_{0}}^{\beta}$ is relatively compact in $H_{\alpha}[0,1]$ for any $0<\alpha<\beta \leq 1$. Furthermore, $B_{r_{0}}^{\beta}$ is a compact subset in $H_{\alpha}[0,1]$.

In the sequel, we will demonstrate that the operator $F$ is continuous on $B_{r_{0}}^{\beta}$ with respect to the norm $\|\cdot\|_{\alpha}$, where $0<\alpha<\beta \leq 1$.

Let $y \in B_{r_{0}}^{\beta}$ be an arbitrary point in $B_{r_{0}}^{\beta}$. Then, we get:

$$
\begin{aligned}
& (F x)(t)-(F y)(t)-((F x)(s)-(F y)(s)) \\
= & p(t)+x(t) \int_{0}^{1} k(t, \tau)(T x)(\tau) d \tau \\
& -p(t)-y(t) \int_{0}^{1} k(t, \tau)(T y)(\tau) d \tau \\
& -p(s)-x(s) \int_{0}^{1} k(s, \tau)(T x)(\tau) d \tau \\
& +p(s)+y(s) \int_{0}^{1} k(s, \tau)(T y)(\tau) d \tau
\end{aligned}
$$

for any $x \in B_{r_{0}}^{\beta}$ and $t, s \in[0,1]$. Equality (11) can be rewritten as:

$$
\begin{aligned}
& (F x)(t)-(F y)(t)-((F x)(s)-(F y)(s)) \\
= & x(t) \int_{0}^{1} k(t, \tau)(T x)(\tau) d \tau-y(t) \int_{0}^{1} k(t, \tau)(T x)(\tau) d \tau \\
& +y(t) \int_{0}^{1} k(t, \tau)(T x)(\tau) d \tau-y(t) \int_{0}^{1} k(t, \tau)(T y)(\tau) d \tau \\
& -x(s) \int_{0}^{1} k(s, \tau)(T x)(\tau) d \tau+y(s) \int_{0}^{1} k(s, \tau)(T x)(\tau) d \tau \\
& -y(s) \int_{0}^{1} k(s, \tau)(T x)(\tau) d \tau+y(s) \int_{0}^{1} k(s, \tau)(T y)(\tau) d \tau .
\end{aligned}
$$

By (12), we have:

$$
\begin{aligned}
& (F x)(t)-(F y)(t)-((F x)(s)-(F y)(s)) \\
= & (x(t)-y(t)) \int_{0}^{1} k(t, \tau)(T x)(\tau) d \tau \\
& +y(t) \int_{0}^{1} k(t, \tau)((T x)(\tau)-(T y)(\tau)) d \tau \\
& -(x(s)-y(s)) \int_{0}^{1} k(s, \tau)(T x)(\tau) d \tau \\
& -y(s) \int_{0}^{1} k(s, \tau)((T x)(\tau)-(T y)(\tau)) d \tau .
\end{aligned}
$$


(13) yields the following equality:

$$
\begin{aligned}
& ((F x)(t)-(F y)(t))-((F x)(s)-(F y)(s)) \\
= & {[(x(t)-y(t))-(x(s)-y(s))] \int_{0}^{1} k(t, \tau)(T x)(\tau) d \tau } \\
& +(x(s)-y(s)) \int_{0}^{1}(k(t, \tau)-k(s, \tau))(T x)(\tau) d \tau \\
& +(y(t)-y(s)) \int_{0}^{1} k(t, \tau)((T x)(\tau)-(T y)(\tau)) d \tau \\
& +y(s) \int_{0}^{1}(k(t, \tau)-k(s, \tau))((T x)(\tau)-(T y)(\tau)) d \tau .
\end{aligned}
$$

Hence, taking into account (14), we can write:

$$
\begin{aligned}
& \frac{|(F x)(t)-(F y)(t)-((F x)(s)-(F y)(s))|}{\left.|t-s|^{\alpha}\right)} \\
\leq & \frac{|(x(t)-y(t))-(x(s)-y(s))|}{\mid t-s \alpha^{\alpha}} \int_{0}^{1}|k(t, \tau) \|(T x)(\tau)| d \tau \\
& +\frac{|x(s)-y(s)|}{|t-s|^{\alpha}} \int_{0}^{1}|k(t, \tau)-k(s, \tau) \|(T x)(\tau)| d \tau \\
& +\frac{|y(t)-y(s)|}{|t-s|^{\alpha}} \int_{0}^{1}|k(t, \tau) \|(T x)(\tau)-(T y)(\tau)| d \tau \\
& +\frac{|y(s)|}{|t-s|^{\alpha}} \int_{0}^{1}|k(t, \tau)-k(s, \tau) \|(T x)(\tau)-(T y)(\tau)| d \tau \\
\leq \quad & \|x-y\|_{\alpha}\|T x\|_{\infty} K+\|x-y\|_{\infty}\|T x\|_{\infty} \int_{0}^{1} k_{\beta}|t-s|^{\beta-\alpha} d \tau \\
& +\|y\|_{\alpha}\|T x-T y\|_{\infty} K+\|y\|_{\infty}\|T x-T y\|_{\infty} \int_{0}^{1} k_{\beta}|t-s|^{\beta-\alpha} d \tau \\
\leq & K\|x-y\|_{\alpha}\|T x\|_{\infty}+k_{\beta}\|x-y\|_{\alpha}\|T x\|_{\infty} \\
& +K\|y\|_{\alpha}\|T x-T y\|_{\infty}+k_{\beta}\|y\|_{\alpha}\|T x-T y\|_{\infty} \\
\leq \quad & K f\left(\|x\|_{\beta}\right)\|x-y\|_{\alpha}+k_{\beta} f\left(\|x\|_{\beta}\right)\|x-y\|_{\alpha} \\
& +K\|y\|_{\alpha}\|T x-T y\|_{\infty}+k_{\beta}\|y\|_{\alpha}\|T x-T y\|_{\infty} \\
= & \left(K+k_{\beta}\right) f\left(\|x\|_{\beta}\right)\|x-y\|_{\alpha}+\left(K+k_{\beta}\right)\|y\|_{\alpha}\|T x-T y\|_{\infty}
\end{aligned}
$$

for all $t, s \in[0,1]$ with $t \neq s$. Besides, for $x, y \in B_{r_{0}}^{\beta}$, we obtain the following inequality:

$$
\begin{aligned}
& |(F x)(0)-(F y)(0)| \\
= & \mid p(0)+x(0) \int_{0}^{1} k(0, \tau)(T x)(\tau) d \tau \\
& -p(0)-y(0) \int_{0}^{1} k(0, \tau)(T y)(\tau) d \tau \mid \\
= & \mid x(0) \int_{0}^{1} k(0, \tau)(T x)(\tau) d \tau-y(0) \int_{0}^{1} k(0, \tau)(T x)(\tau) d \tau \\
& +y(0) \int_{0}^{1} k(0, \tau)(T x)(\tau) d \tau-y(0) \int_{0}^{1} k(0, \tau)(T y)(\tau) d \tau \mid \\
= & \mid(x(0)-y(0)) \int_{0}^{1} k(0, \tau)(T x)(\tau) d \tau \\
& +y(0) \int_{0}^{1} k(0, \tau)((T x)(\tau)-(T y)(\tau)) d \tau \mid \\
\leq \quad & \|x-y\|_{\infty} K\|T x\|_{\infty}+\|y\|_{\infty} K\|T x-T y\|_{\infty} \\
\leq & K\|x-y\|_{\alpha}\|T x\|_{\infty}+K\|y\|_{\alpha}\|T x-T y\|_{\infty} \\
\leq & K f\left(\|x\|_{\beta}\right)\|x-y\|_{\alpha}+K\|y\|_{\alpha}\|T x-T y\|_{\infty} .
\end{aligned}
$$

From (15) and (16), we have that:

$$
\begin{aligned}
& \|F x-F y\|_{\alpha} \\
= & |(F x-F y)(0)|+H_{F x-F y}^{\alpha} \\
= & |(F x)(0)-(F y)(0)| \\
& +\sup \left\{\frac{|(F x)(t)-(F y)(t)-((F x)(s)-(F y)(s))|}{|t-s|^{\alpha}}: t, s \in[0,1] \text { and } t \neq s\right\} \\
\leq & \left(2 K+k_{\beta}\right) f\left(\|x\|_{\beta}\right)\|x-y\|_{\alpha}+\left(2 K+k_{\beta}\right)\|y\|_{\alpha}\|T x-T y\|_{\infty} .
\end{aligned}
$$


Moreover, since $\|y\|_{\alpha} \leq\|y\|_{\beta} \leq r_{0}$ and $f\left(\|x\|_{\beta}\right) \leq f\left(r_{0}\right)$, we derive from (17) that the following inequality holds:

$$
\|F x-F y\|_{\alpha} \leq\left(2 K+k_{\beta}\right) f\left(r_{0}\right)\|x-y\|_{\alpha}+\left(2 K+k_{\beta}\right) r_{0}\|T x-T y\|_{\infty} .
$$

Since the operator $T: H_{\beta}[0,1] \rightarrow C[0,1]$ is continuous on $H_{\beta}[0,1]$ with respect to the norm $\|\cdot\|_{\alpha^{\prime}}$ it is also continuous at the point $y \in B_{r_{0}}^{\beta}$. Let us take an arbitrary $\varepsilon>0$. Since the operator $T$ is continuous at the point $y \in B_{r_{0}}^{\beta}$, there exists $\delta>0$ such that the inequality:

$$
\|T x-T y\|_{\infty}<\frac{\varepsilon}{2\left(2 K+k_{\beta}\right) r_{0}}
$$

is satisfied for all $x \in B_{r_{0}}^{\beta}$, where $\|x-y\|_{\alpha}<\delta$ and:

$$
0<\delta<\frac{\varepsilon}{2\left(2 K+k_{\beta}\right) f\left(r_{0}\right)} .
$$

Then, taking into account (18), we derive the following inequality:

$$
\begin{aligned}
\|F x-F y\|_{\alpha} & \leq\left(2 K+k_{\beta}\right) f\left(r_{0}\right)\|x-y\|_{\alpha}+\left(2 K+k_{\beta}\right) r_{0}\|T x-T y\|_{\infty} \\
& <\frac{\varepsilon}{2}+\frac{\varepsilon}{2} \\
& =\varepsilon .
\end{aligned}
$$

As a result, we infer that the operator $F$ is continuous at the point $y \in B_{r_{0}}^{\beta}$. Because $y$ was chosen arbitrarily, we deduce that $F$ is continuous on $B_{r_{0}}^{\beta}$ with respect to the norm $\|\cdot\|_{\alpha}$. As $B_{r_{0}}^{\beta}$ is compact in $H_{\alpha}[0,1]$, from the classical Schauder fixed point theorem, we get the desired result.

\section{Examples}

In this part, we conclude the article by presenting two examples that illustrate the generality and efficiency of our results.

Example 1. Let us consider the following quadratic integral equation:

$$
x(t)=\sqrt[6]{q \cos t+\hat{q}}+x(t) \int_{0}^{1} \sqrt[5]{m t^{2}+\tau} \sin x^{2}(\tau) d \tau, \quad t \in I=[0,1] .
$$

Here, $q, \hat{q}$ and $m$ are the suitable nonnegative constants to be determined such that Conditions (i)-(iv) of Theorem 2 hold.

Set $p(t)=\sqrt[6]{q \cos t+\hat{q}}$ and $k(t, \tau)=\sqrt[5]{m t^{2}+\tau}$ for all $t, \tau \in[0,1]$.

It is easily seen that:

$$
\begin{aligned}
|p(t)-p(s)| & =|\sqrt[6]{q \cos t+\hat{q}}-\sqrt[6]{q \cos s+\hat{q}}| \\
& \leq|\sqrt[6]{q \cos t+\hat{q}-q \cos s-\hat{q}}| \\
& \leq \sqrt[6]{q|\cos t-\cos s|} \\
& \leq \sqrt[6]{2 q\left|\sin \left(\frac{t+s}{2}\right)\right|\left|\sin \left(\frac{t-s}{2}\right)\right|} \\
& \leq \sqrt[6]{q} \sqrt[6]{|t-s|} \\
& =\sqrt[6]{q}|t-s|^{\frac{1}{6}}
\end{aligned}
$$


for all $t, s \in[0,1]$. This says that $p \in H_{\frac{1}{6}}[0,1]$ and, moreover, $H_{p}^{\frac{1}{6}}=\sqrt[6]{q}$. Therefore, we can take the constants $\alpha$ and $\beta$ as $0<\alpha<\frac{1}{6}$ and $\beta=\frac{1}{6}$. Therefore, Assumption (i) of Theorem 2 holds. Note that:

$$
\begin{aligned}
\|p\|_{\frac{1}{6}} & =|p(0)|+\sup \left\{\frac{|p(t)-p(s)|}{|t-s|^{\frac{1}{6}}}: t, s \in[0,1] \text { and } t \neq s\right\} \\
& =|p(0)|+H_{p}^{\frac{1}{6}}=\sqrt[6]{q+\hat{q}}+\sqrt[6]{q} .
\end{aligned}
$$

Further, we have:

$$
\begin{aligned}
|k(t, \tau)-k(s, \tau)| & =\left|\sqrt[5]{m t^{2}+\tau}-\sqrt[5]{m s^{2}+\tau}\right| \\
& \leq\left|\sqrt[5]{m\left(t^{2}-s^{2}\right)}\right| \\
& =\sqrt[5]{m} \sqrt[5]{\left|\left(t^{2}-s^{2}\right)\right|} \\
& =\sqrt[5]{m} \sqrt[5]{|t-s|} \sqrt[5]{|t+s|} \\
& \leq \sqrt[5]{2 m}|t-s|^{\frac{1}{6}}|t-s|^{\frac{1}{30}} \\
& \leq \sqrt[5]{2 m}|t-s|^{\frac{1}{6}}
\end{aligned}
$$

for all $t, s, \tau \in[0,1]$. Assumption (ii) of Theorem 2 holds with $k_{\beta}=k_{\frac{1}{6}}=\sqrt[5]{2 m}$.

Since $(T x)(\tau)=\sin x^{2}(\tau)$ and:

$$
\left|\sin x^{2}(\tau)\right| \leq\left|x^{2}(\tau)\right|=\left|x(\tau)\|x(\tau) \mid \leq\| x\left\|_{\infty}^{2} \leq\right\| x \|_{\beta}^{2}\right.
$$

for all $x \in H_{\beta}[0,1]$ and $\tau \in[0,1]$, the inequality:

$$
\|T x\|_{\infty}=\sup _{\tau \in[0,1]}\left|\sin x^{2}(\tau)\right| \leq\|x\|_{\beta}^{2}
$$

holds. Therefore, we can choose the function $f: \mathbb{R}_{+} \rightarrow \mathbb{R}_{+}$as $f(x)=x^{2}$. This function is non-decreasing and satisfies the inequality in Assumption (iii).

We will show that the operator $T: H_{\beta}[0,1] \rightarrow C[0,1]$ is continuous on $H_{\beta}[0,1]$ with respect to the norm $\|\cdot\|_{\alpha}$. Let us take $x, y \in H_{\beta}[0,1]$ and $\tau \in[0,1]$.

It is clear that:

$$
\begin{aligned}
\left|\sin x^{2}(\tau)-\sin y^{2}(\tau)\right| & \leq\left|x^{2}(\tau)-y^{2}(\tau)\right| \\
& =|x(\tau)-y(\tau)||x(\tau)+y(\tau)| \\
& =|x(\tau)-y(\tau)||x(\tau)-y(\tau)+2 y(\tau)| \\
& \leq|x(\tau)-y(\tau)|(|x(\tau)-y(\tau)|+2|y(\tau)|) \\
& \leq\|x-y\|_{\infty}\left(\|x-y\|_{\infty}+2\|y\|_{\infty}\right) \\
& \leq\|x-y\|_{\alpha}\left(\|x-y\|_{\alpha}+2\|y\|_{\alpha}\right)
\end{aligned}
$$

and:

$$
\|T x-T y\|_{\infty} \leq \sup _{\tau \in[0,1]}\left|\sin x^{2}(\tau)-\sin y^{2}(\tau)\right| \leq\|x-y\|_{\alpha}\left(\|x-y\|_{\alpha}+2\|y\|_{\alpha}\right)
$$


Now, we will show that $T$ is continuous at the point $y \in H_{\beta}[0,1]$ with respect to the norm $\|\cdot\|_{\alpha}$. Let us take an arbitrary $\varepsilon>0$. Then, there exists the positive number $\delta$ such that $\|x-y\|_{\alpha}<\delta$ and the inequality:

$$
\|T x-T y\|_{\infty}<\delta\left(\delta+2\|y\|_{\alpha}\right)<\varepsilon
$$

is satisfied for all $x \in H_{\beta}[0,1]$, where $0<\delta<\sqrt{\|y\|_{\alpha}^{2}+\varepsilon}-\|y\|_{\alpha}$. Therefore, we can choose the positive number $\delta$ as $\delta=\frac{1}{2} \sqrt{\|y\|_{\alpha}^{2}+\varepsilon}-\|y\|_{\alpha}$. As a result, we infer that the operator $T$ is continuous at the point $y \in B_{r_{0}}^{\beta}$. Since $y$ was chosen arbitrarily, we deduce that $T$ is continuous on $H_{\beta}[0,1]$ with respect to the norm $\|\cdot\|_{\alpha}$.

Further, we can calculate that:

$$
\begin{aligned}
\sup \left\{\int_{0}^{1}|k(t, \tau)| d \tau: t \in[0,1]\right\} & =\sup \left\{\int_{0}^{1}\left|\sqrt[5]{m t^{2}+\tau}\right| d \tau: t \in[0,1]\right\} \\
& =\sup \left\{\frac{5}{6}\left(\sqrt[5]{\left(m t^{2}+1\right)^{6}}-\sqrt[5]{\left(m t^{2}\right)^{6}}\right): t \in[0,1]\right\} \\
& \leq \sup \left\{\frac{5}{6} \sqrt[5]{\left(m t^{2}+1\right)^{6}}: t \in[0,1]\right\} \\
& =\frac{5}{6} \sqrt[5]{(m+1)^{6}} \\
& \leq \sqrt[5]{(m+1)^{6}} \\
& =K
\end{aligned}
$$

In this case, the inequality appearing in assumption (vi) of Theorem 2 takes the following form:

$$
\|p\|_{\frac{1}{6}}+\left(2 K+k_{\beta}\right) r f(r) \leq r
$$

which is equivalent to:

$$
\sqrt[6]{q}+\sqrt[6]{q+\hat{q}}+\left(2 \sqrt[5]{(m+1)^{6}}+\sqrt[5]{2 m}\right) r r^{2} \leq r
$$

There exists a positive number $r_{0}$ satisfying (20) provided that the constants $q, \hat{q}$ and $m$ are chosen as suitable.

For example, if one chose $q=\frac{1}{10^{18}}, \hat{q}=0$ and $m=\frac{1}{2^{16}}$, then the inequality:

$$
\frac{2}{10^{3}}+\left(2 \sqrt[5]{\left(\frac{1}{2^{16}}+1\right)^{6}}+0.125\right) r^{3} \leq r
$$

holds for $r=r_{0}=\frac{1}{10}$. Therefore, using Theorem 2, we infer that there is at least one solution $x$ of Equation (19) in the space $H_{\alpha}[0,1]$ with $0<\alpha<\frac{1}{6}$.

Example 2. Let us consider the following quadratic integral equation:

$$
x(t)=\frac{1}{10^{6}} \arctan \sqrt[3]{t+\ln q}+x(t) \int_{0}^{1} \sqrt[3]{m \sin t+\tau} \sqrt{|x(\tau)|} d \tau, \quad t \in I=[0,1],
$$

where $q$ and $m$ are the suitable positive constants to be selected for which Conditions (i)-(iv) of Theorem 2 hold. Set $p(t)=\frac{1}{10^{6}} \arctan \sqrt[3]{t+\ln q}$ and $k(t, \tau)=\sqrt[3]{m \sin t+\tau}$ for all $t, \tau \in[0,1]$. 
It is obvious that the inequality:

$$
\begin{aligned}
|p(t)-p(s)| & =\left|\frac{1}{10^{6}} \arctan \sqrt[3]{t+\ln q}-\frac{1}{10^{6}} \arctan \sqrt[3]{s+\ln q}\right| \\
& \leq\left|\frac{1}{10^{6}} \arctan (\sqrt[3]{t+\ln q}-\sqrt[3]{s+\ln q})\right| \\
& =\frac{1}{10^{6}}|\sqrt[3]{t+\ln q}-\sqrt[3]{s+\ln q}| \\
& \leq \frac{1}{10^{6}}|\sqrt[3]{t+\ln q-s-\ln q}| \\
& \leq \frac{1}{10^{6}} \sqrt[3]{|t-s|} \\
& =\frac{1}{10^{6}}|t-s|^{\frac{1}{3}}
\end{aligned}
$$

holds for all $t, s \in[0,1]$. Therefore, $p \in H_{\frac{1}{3}}[0,1]$ and $H_{p}^{\frac{1}{3}}=\frac{1}{10^{6}}$. Hence, the constants $\alpha$ and $\beta$ can be taken as $0<\alpha<\frac{1}{3}$ and $\beta=\frac{1}{3}$.

Therefore, Assumption (i) of Theorem 2 is satisfied. Note that:

$$
\begin{aligned}
\|p\|_{\frac{1}{3}} & =|p(0)|+\sup \left\{\frac{|p(t)-p(s)|}{|t-s|^{\frac{1}{3}}}: t, s \in[0,1] \text { and } t \neq s\right\} \\
& =|p(0)|+H_{p}^{\frac{1}{3}}=\frac{1}{10^{6}}|\arctan \sqrt[3]{\ln q}|+\frac{1}{10^{6}} .
\end{aligned}
$$

Further, we have:

$$
\begin{aligned}
|k(t, \tau)-k(s, \tau)| & =|\sqrt[3]{m \sin t+\tau}-\sqrt[3]{m \sin s+\tau}| \\
& \leq|\sqrt[3]{m(\sin t-\sin s)}| \\
& \leq \sqrt[3]{2 m\left|\cos \left(\frac{t+s}{2}\right)\right|\left|\sin \left(\frac{t-s}{2}\right)\right|} \\
& \leq \sqrt[3]{m}|t-s|^{\frac{1}{3}}
\end{aligned}
$$

for all $t, s, \tau \in[0,1]$. Assumption (ii) of Theorem 2 is satisfied with $k_{\beta}=k_{\frac{1}{3}}=\sqrt[3]{m}$.

Since $(T x)(\tau)=\sqrt{|x(\tau)|}$ and:

$$
\sqrt{|x(\tau)|} \leq \sqrt{\|x\|_{\infty}} \leq \sqrt{\|x\|_{\beta}}
$$

for all $x \in H_{\beta}[0,1]$ and $\tau \in[0,1]$, the inequality:

$$
\|T x\|_{\infty}=\sup _{\tau \in[0,1]}|\sqrt{|x(\tau)|}| \leq \sqrt{\|x\|_{\beta}}
$$

holds. Therefore, we can choose the function $f: \mathbb{R}_{+} \rightarrow \mathbb{R}_{+}$as $f(x)=\sqrt{x}$. This function is non-decreasing and satisfies the inequality in Assumption (iii).

We will show that the operator $T: H_{\beta}[0,1] \rightarrow C[0,1]$ is continuous on $H_{\beta}[0,1]$ with respect to the norm $\|\cdot\|_{\alpha}$. Let us take $x, y \in H_{\beta}[0,1]$ and $\tau \in[0,1]$. It is certain that:

$$
|\sqrt{|x(\tau)|}-\sqrt{|y(\tau)|}| \leq \sqrt{|x(\tau)-y(\tau)|} \leq \sqrt{\|x-y\|_{\infty}} \leq \sqrt{\|x-y\|_{\alpha}}
$$


and:

$$
\|T x-T y\|_{\infty} \leq \sup _{\tau \in[0,1]}|\sqrt{|x(\tau)|}-\sqrt{|y(\tau)|}| \leq \sqrt{\|x-y\|_{\alpha}}
$$

Now, we will show that $T$ is continuous at the point $y \in H_{\beta}[0,1]$ with respect to the norm $\|\cdot\|_{\alpha}$. Let us take an arbitrary $\varepsilon>0$. Then, there exists the positive number $\delta$ such that $\|x-y\|_{\alpha}<\delta$ and the inequality:

$$
\|T x-T y\|_{\infty} \leq \sqrt{\|x-y\|_{\alpha}}<\varepsilon
$$

is satisfied for all $x \in H_{\beta}[0,1]$. Here, we can choose the positive number $\delta$ as $\delta=\varepsilon^{2}$.

As a result, we infer that the operator $T$ is continuous at the point $y \in B_{r_{0}}^{\beta}$. Since $y$ was chosen arbitrarily, we deduce that $T$ is continuous on $H_{\beta}[0,1]$ with respect to the norm $\|\cdot\|_{\alpha}$.

Further, we can calculate that:

$$
\begin{aligned}
& \sup \left\{\int_{0}^{1}|k(t, \tau)| d \tau: t \in[0,1]\right\} \\
= & \sup \left\{\int_{0}^{1}|\sqrt[3]{m \sin t+\tau}| d \tau: t \in[0,1]\right\} \\
= & \sup \left\{\frac{1}{3}\left(\sqrt[3]{(m \sin t+1)^{4}}-\sqrt[3]{(m \sin t)^{4}}\right): t \in[0,1]\right\} \\
\leq & \sup \left\{\frac{1}{3} \sqrt[3]{(m \sin t+1)^{4}}: t \in[0,1]\right\} \\
\leq & \frac{1}{3} \sqrt[3]{(m+1)^{4}} \\
\leq & \sqrt[3]{(m+1)^{4}} \\
= & K .
\end{aligned}
$$

In this case, the inequality appearing in Assumption (vi) of Theorem 2 takes the following form:

$$
\|p\|_{\frac{1}{3}}+\left(2 K+k_{\beta}\right) r f(r) \leq r
$$

which is equivalent to:

$$
\frac{1}{10^{6}}(|\arctan \sqrt[3]{\ln q}|+1)+\left(2 \sqrt[3]{(m+1)^{4}}+\sqrt[3]{m}\right) r \sqrt{r} \leq r
$$

There exists a positive number $r_{0}$ satisfying (22) for chosen suitable constants $q$ and $m$.

For example, if one chooses $q=1$ and $m=\frac{1}{5^{12}}$, then the inequality:

$$
\frac{1}{10^{6}}+\left(2 \sqrt[3]{\left(1+\frac{1}{5^{12}}\right)^{4}}+0.0016\right) r^{\frac{3}{2}} \leq r
$$

holds for $r=r_{0}=\frac{1}{10^{4}}$. Therefore, using Theorem 2, we infer that there is at least one solution $x$ of Equation (21) in the space $H_{\alpha}[0,1]$ with $0<\alpha<\frac{1}{3}$.

\section{Conclusions}

In this paper, we have investigated the existence of solutions of the integral Equation (4). It should be noted that Equation (4) is more general than many equations considered up to now. For example, it includes the equations examined in previous studies [4-6]. That is, if we take the operator $T$ as 
$(T x)(\tau)=x(\tau)$, we obtain the integral Equation (1) in [4] with $a=0$ and $b=1$. On the other hand, if we take $(T x)(\tau)=x(r(\tau))$, we have the integral Equation (2) in [5]. Further, if we take $(T x)(\tau)=\max _{\eta \in[0, r(\tau)]}|x(\eta)|$, we have the integral Equation (3) in [6].

Author Contributions: Writing, original draft, M.T.E. and H.F. Writing, review and editing, M.T.E. and H.F.

Acknowledgments: M.T.E. was supported by the Scientific and Technological Research Council of Turkey (TUBITAK Programme, 2228-B).

Conflicts of Interest: The authors declare no conflict of interest.

\section{References}

1. Kulenovic, M.R.S. Oscillation of the Euler differential equation with delay. Czech. Math. J. 1995, 45, 1-16.

2. Mureşan, V. On a class of Volterra integral equations with deviating argument. Stud. Univ. Babes-Bolyai Math. 1999, 44, 47-54.

3. Mureşan, V. Volterra integral equations with iterations of linear modification of the argument. Novi Sad J. Math. 2003, 33, 1-10.

4. Banaś, J.; Nalepa, R. On the space of functions with growths tempered by a modulus of continuity and its applications. J. Funct. Spaces Appl. 2013. [CrossRef]

5. Caballero, J.; Abdalla, M.; Sadarangani, K. Solvability of a quadratic integral equation of fredholm type in Hölder spaces. Electron. J. Differ. Equ. 2014, 31, 1-10. [CrossRef]

6. Caballero, M.J.; Nalepa, R.; Sadarangani, K. Solvability of a quadratic integral equation of Fredholm type with Supremum in Hölder Spaces. J. Funct. Spaces Appl. 2014. [CrossRef]

7. Schauder, J. Der Fixpunktsatz in Funktionalriiumen. Stud. Math. 1930, 2, 171-180. [CrossRef]

8. López, B.; Harjani, J.; Sadaragani, K. Existence of positive solutions in the space of Lipschitz functions to a class of fractional differential equations of arbitrary order. Racsam 2018, 112, 1281-1294. [CrossRef]

9. Bacotiu, C. Volterra-Fredholm nonlinear systems with modified argument via weakly Picard operators theory. Carpathian J. Math. 2008, 24, 1-19.

10. Benchohra, M.; Darwish, M.A. On unique solvability of quadratic integral equations with linear modification of the argument. Miskolc Math. Notes 2009, 10, 3-10.

11. Dobritoiu, M. Analysis of a nonlinear integral equation with modified argument from physics. Int. J. Math. Models Methods Appl. Sci. 2008, 2, 403-412.

12. Kato, T.; Mcleod, J.B. The functional-differential equation $y^{\prime}(x)=a y(\lambda x)+b y(x)$. Bull. Am. Math. Soc. 1971, 77, 891-937.

13. Lauran, M. Existence results for some differential equations with deviating argument. Filomat 2011, 25, 21-31. [CrossRef]

14. Mureşan, V. A functional-integral equation with linear modification of the argument via weakly Picard operators. Fixed Point Theory 2008, 9, 189-197.

15. Mureşan, V. A Fredholm-Volterra integro-differential equation with linear modification of the argument. J. Appl. Math. 2010, 3, 147-158.

16. Agarwal, R.P.; O'Regan, D. Infinite Interval Problems for Differential, Difference and Integral Equations; Springer: Dordrecht, The Netherlands, 2001; ISBN 978-94-015-9171-3.

17. Agarwal, R.P.; O'Regan, D.; Wong, P.J.Y. Positive Solutions of Differential, Difference and Integral Equations; Springer: Dordrecht, The Netherlands, 1999.

18. Case, K.M.; Zweifel, P.F. Linear Transport Theory; Addison Wesley: Reading, MA, USA, 1967.

19. Chandrasekhar, S. Radiative Transfer; Dover Publications: New York, NY, USA, 1960.

20. Hu, S.; Khavani, M.; Zhuang, W. Integral equations arising in the kinetic theory of gases. J. Appl. Anal. 1989, 34, 261-266. [CrossRef]

21. Kelly, C.T. Approximation of solutions of some quadratic integral equations in transport theory. J. Integral Equ. 1982, 4, 221-237.

22. Banas, J.; Lecko, M.; El-Sayed, W.G. Existence theorems of some quadratic integral equation. J. Math. Anal. Appl. 1998, 222, 276-285. [CrossRef]

23. Banas, J.; Caballero, J.; Rocha, J.; Sadarangani, K. Monotonic solutions of a class of quadratic integral equations of Volterra type. Comput. Math. Appl. 2005, 49, 943-952. [CrossRef] 
24. Caballero, J.; Rocha, J.; Sadarangani, K. On monotonic solutions of an integral equation of Volterra type. J. Comput. Appl. Math. 2005, 174, 119-133. [CrossRef]

25. Darwish, M.A. On solvability of some quadratic functional-integral equation in Banach algebras. Commun. Appl. Anal. 2007, 11, 441-450.

26. Darwish, M.A.; Ntouyas, S.K. On a quadratic fractional Hammerstein-Volterra integral equations with linear modification of the argument. Nonlinear Anal. Theory Methods Appl. 2011, 74, 3510-3517. [CrossRef]

27. Darwish, M.A. On quadratic integral equation of fractional orders. J. Math. Anal. Appl. 2005, 311, 112-119. [CrossRef]

28. Agarwal, R.P.; Banas, J.; Banas, K.; O’Regan, D. Solvability of a quadratic Hammerstein integral equation in the class of functions having limits at infinity. J. Integral Equ. Appl. 2011, 23, 157-181. [CrossRef]

29. Marin, M. An approach of a heat-flux dependent theory for micropolar porous media. Meccanica 2016, 51, 1127-1133. [CrossRef]

30. Marin, M. Some estimates on vibrations in thermoelasticity of dipolar bodies. J. Vib. Control 2010, 16, 33-47. [CrossRef]

31. Caballero, J.; Darwish, M.A.; Sadarangani, K. Positive Solutions in the Space of Lipschitz Functions for Fractional Boundary Value Problems with Integral Boundary Conditions. Mediterr. J. Math. 2017. [CrossRef]

32. Cabrera, I.; Harjani, J.; Sadarangani, K. Existence and Uniqueness of Solutions for a Boundary Value Problem of Fractional Type with Nonlocal Integral Boundary Conditions in Hölder Spaces. Mediterr. J. Math. 2018. [CrossRef]

(C) 2018 by the authors. Licensee MDPI, Basel, Switzerland. This article is an open access article distributed under the terms and conditions of the Creative Commons Attribution (CC BY) license (http:/ / creativecommons.org/licenses/by/4.0/). 